\title{
International Medical Graduates: A Cohort Study of Key Informant Perspectives
}

\author{
Daniel R Terry*, Quynh Lê \\ Department of Rural Health, University of Tasmania, Locked Bag 1372, Launceston, Tasmania 7250, Australia \\ *Corresponding Author: Daniel.Terry@utas.edu.au
}

Copyright (C) 2013 Horizon Research Publishing All rights reserved.

\begin{abstract}
An Australian wide shortage of doctors has led to an increased reliance on International Medical Graduate (IMG) recruitment. However, there is very little research regarding the acculturation of IMGs as they reside in the rural Tasmanian context. This paper explores from the perspective of IMG informants, the experiences and challenges of IMGs living and working in rural and remote Tasmania and how it informs the acculturation process. It identifies the barriers facing IMGs as they work and live in Tasmanian communities. It is part of a larger doctoral study looking at IMGs in Tasmania. The study adopted a qualitative research approach. Twenty-three semi-structured interviews were conducted with IMG informants in the North, South and North West of Tasmania. Informants were recruited through purposive snowball sampling. The findings indicate that Tasmanian based IMGs encounter both professional and social challenges. These challenges include vulnerability due to fear of job security, and loss of status, discrimination and communication challenges within the workplace. Additional challenges include spouse employment, obtaining high quality academic access for children, and cultural and religious connectivity. These challenges influence IMGs and their families to stay or relocate. In addition, a number of identified needs have been identified to improve retention of IMGs within Tasmania. The study adds to existing knowledge and identifies three factors which further determine the retention of IMGs. These factors include, job opportunities, vocational training and greater commensurate support provided to IMGs and their families.
\end{abstract}

Keywords International Medical Graduates, Rural, Professional Challenges, Social Challenges, Acculturation

\section{Introduction}

An Australian wide shortage of doctors has led to an increased reliance on the recruitment of International Medical Graduates (IMGs) This reliance on IMGs is not specific to Australia but is a world-wide phenomenon. [1,2]
IMGs represent $24 \%$ of all medical practitioners in Australia [3], whereas a report by the Department of Health and Ageing (DoHA) states this number is as high as 39\% across Australia and as high as $52 \%$ in a number of rural and remote areas. [4] Under current Australian policy mandates, IMGs are placed in rural and remote 'areas of need' where identified gaps or shortages in the local medical workforce exist. [4] These areas of need, determined by State and Territory Governments allow an IMG to be conditionally registered to receive an exemption on Medicare provider number restrictions. This allows the IMG to claim Medicare rebates for providing professional services. [4-7]

Currently, the Australian Medical Council (AMC) assesses IMGs who are seeking to practise medicine in Australia. There are four alternate pathways for registration in Australia, the specialist recognition competent authority or the standard pathway which follows two streams which consist of the completion of a workplace based assessment or passing a clinical examination. [8] For each individual, it is dependent on where the IMG has obtained their primary training and what current qualification they possess. In most cases, the AMC rigorously verifies an IMGs credentials, the country of origin's examining and accrediting processes and administers a multiple choice question (MCQ) exam, of which $84 \%$ of those who sit, complete within the first two attempts. [4,9] As such the AMC demonstrated, 61.41\% $(n=2353)$ of all examination attempts in 2010 were made by IMGs from Bangladesh, Sri Lanka, Pakistan, Myanmar, Philippines, Iran and India. [9] Following the MCQ and provisional medical registration, either a clinical interview, workplace based assessment or a clinical examination is conducted. $[8,10,11]$

Many concerns regarding immigration, appropriate support and ongoing examination processes have been expressed by IMGs, most of which arrive as temporary residents and are sponsored by an employer to work in Australia on limited registration. [4,12] However, very little research within Australian or internationally has been undertaken to examine the quality of life and social needs of IMGs and their families as crucial factors impacting the integration and acculturation as IMGs reside in rural and remote areas. 


\subsection{The Literature}

A review of the literature revealed there were twenty-one articles which were in some capacity aimed at investigating and understanding the professional experiences, social support, acculturation and retention of IMGs. Ten were international studies while the remaining 11 studies were conducted in Australia. [13] Two of the ten international studies examined the personal challenges of migration of both IMGs and their family in terms of being part of their new community. $[14,15]$ The remainder was focused on the employment context of retention and acculturation. For example, a study conducted by Atri, et al. [16], examined the effects of social support and acculturation of IMGs on their mental health and wellbeing and their capacity to be professionally integrated within the work setting in the US. What remained lacking in many of the studies was an analysis of IMGs and their families living in rural or remote settings and the factors which affect IMGs social integration. Two studies, were based in Canada [15,17], three were conducted in New Zealand $[14,18,19]$, one study was UK based [20], while the remaining three were in the US, $[16,21,22]$ and lastly, one study was based in the US, but specifically examined former Soviet Union doctors working in the USA, Israel and Canada. [23]

Similarly, within the Australian context, there have been eleven commensurate studies which relate to this current study. Those identified studies were conducted in various states of rural Australia. This included studies in New South Wales (NSW) [24], Queensland (QLD) [25], two in South Australia (SA) [6,26], three in Victoria (Vic) [27-29] and one in Western Australia (WA) [30]. In addition, there was a study which covered IMGs in the states of QLD, Northern territory (NT) and WA [31], a study which looked at IMGs Australia wide [32] and one study which examined IMGs in ten rural and remote indigenous contexts, however did not disclose in which states these were conducted. [33]

Ten studies were solely examining IMGs, whereas the eleventh study made comparisons between IMGs and those doctors who had trained in Australia. [24] The eleven identified studies have similar aims and objectives, which have ranged from examining IMGs training, support needs and improving orientation for IMGs to determining the key factors which influence IMGs to stay in rural practice. [24-26,28,32,33] In addition at least four studies also analysed the impact of IMGs working and their families living in rural or remote settings and the factors which affect IMGs professional, cultural and social integration. $[27,29,31,34]$ An additional study was found however, it focused on the acculturation of Vietnamese-born health professionals, which included three doctors, a dentist, a physiotherapist and a nurse who trained in Australian. It "examined the life experiences and acculturation strategies of Vietnamese-born health professionals working and living in rural Australia". [35] Although this study was not focused specifically on IMGs, it was noted as an insightful study due to its resonance with the current study and it was the only one of its kind focused in the experience of IMGs in Tasmania.

A number of key issues were identified within the literature, which relate to professional and social barriers IMGs encounter and are discussed in detail.

\subsubsection{Professional barriers}

Many of the Australian and international IMG studies found the central motivation to migrate in a permanent or temporary capacity was for the lifestyle opportunities and increased prospects for family.[15,29-31,33] However, the motivation for a high proportion of doctors from the Pacific islands of Fiji, Samoa and Tonga to migrate to pacific rim countries was strongly associated with income. [36]

Many IMGs regarded migration as temporary measure yet, for some it becomes a more permanent move, including those who cannot or do not wish to return home due to the risk of violence and political instability.[15,23,29,36,37] According to the Australian Rural and Remote Workforce Agencies Group [38], particular locations in Australia are selected by IMGs to live and work in for two main reasons. This included career opportunities and meeting the educational needs of children. This continued make it difficult to recruit and retain IMGs in some rural and remote areas.[29-31,33]

Furthermore, incongruous placement of IMGs may impact negatively on the doctor, their family, the medical practice and the community. As such, many difficulties and challenges are experienced at the time of recruitment.[27,33,39] This may lead to an insufficient understanding of rural communities, how medicine is practised in Australia and how the Australian culture is at times vastly different to an IMGs own culture.[27,33,39,40]

However this can differ between IMGs and the communities they serve. For example, one US study demonstrated $92 \%$ IMGs in rural Wisconsin felt integrated within their workplace and $62 \%$ of the IMG families felt they were well accepted within the community. [21] Whereas, another US study demonstrated only 30\% of IMGs felt integrated within their workplace.[41]

In addition to incongruous placement, it has been shown that many other issues can be challenging prior to and be problematic once rural placement has occurred. These issues include a loss of status and professional identity $[16,17]$; professional isolation in rural practise; the need to sit further medical examinations; the restrictions on medical practise; accessing provider numbers; and social isolation experienced in rural areas.[25,42]For example, a number of rural communities may have had very little experience with individuals from other cultures and may be less welcoming and mistrust people when cultures, customs and religious beliefs are unfamiliar. [38,43,44]

In addition to the professional challenges IMGs encounter, other Australian and international studies have identified a number of specific professional factors which inhibited successful rural integration. As such, these factors will be highlighted and discussed. 


\section{Immigration, accreditation and financial challenges}

Both temporary and permanent IMGs are confronted by many challenges, such as substantial financial pressures when arriving and relocating to a new country and within rural communities. $[18,19,31]$ These financial burdens IMGs are challenged with impact recruitment and retention in rural areas [38,42]. In addition IMGs face long, complex procedures of immigration, accreditation, and costly annual renewal of provisional registration and training. This complex and an all-consuming process has been reported to leave many feeling alienated, angry and uncertain as they endeavour to enter the medical workforce, particularly in Australia, Canada and New Zealand.[17,19,31,44,45]

\section{A lack of facilities and long hours}

In addition to the financial pressures IMGs face, working in rural communities is indicated to be challenging when attempting to maintain a manageable work-life balance.[27,30,45] Doctors working in small rural and remote communities are faced with long working hours and heavy workload, which often includes substantial on-call commitments.[46] It often means working in facilities which have unfamiliar insufficient and inefficient clinical infrastructure and administrative practises. [27,30,45] This additional pressure in the work-live balance impacts on families and creates a greater burden on spouses who may be caring for a family and managing their own professional obligations.[42,47]

In addition to balancing the work and life in rural contexts, many IMGs experience frustration when preparing for and undertaking further educational study and examinations.[14,18] As many IMGs in rural areas study in isolation, while a number of IMGs have trained in disadvantaged educational situations, with marginal access to western technology.[27,29]

\section{Professional support}

The professional support for IMGs, although studied comprehensively remains critical, as it contributes to IMGs inevitable integration and retention in a rural community. [19,24-27,29,31,33,48,49] Professional support also includes colleagues' clinical support and supervision which is remains a precarious factor in assisting IMGs to feel integrated in the practice setting and community.[16]

For example, IMGs joining rural settings may be faced with AMGs who have animosity toward IMG recruitment which can then challenge workplace relationships and create an environment which is less conducive for both parties [39]. A number of contrasting experiences have been voiced by IMGs, particularly in Australia.[30,50] These accounts include being welcomed by colleagues and their families, to professional isolation from others. For example, the experiences extend from nursing staff and managers building strong and lasting professional relationships, to the provision of refurbished practises in close proximity to the hospital as a means to retain IMGs in the community
[30,50]. The support provided by a practice whether positive or negative assists in the camaraderie or isolation experienced and the ultimate acculturation of IMGs.[27,30] Nevertheless, there are additional non-professional, social barriers which impact on IMGs and their families.

\subsubsection{Social barriers}

Within the literature a number of studies examined the motivations for staying or leaving rural practise which showed non-professional, social barrier had the greatest bearing on the decision of where to practise.[25-27,30,31,51] The most prominent non-professional social barriers include employment for spouse, limited schooling options, physical and social isolation and limited cultural and religious access.[52] Lastly a number of challenges regarding community support were also identified. Each of these key barriers will be discussed in greater detail.

\section{Limited educational and employment options for pouses}

A significant barrier impacting IMG rural acculturation is the challenge of limited education and employment opportunities for an IMGs spouse.[14,26,29,33] One study undertaken by Durey [30], examined the impact of living and working in rural or remote Australian communities on IMGs and spouses. It was shown that adequately addressing the needs of an IMGs spouse is vital for the retention of a doctor and the whole family.

In some cases, a spouse may be working in professional, well paid positions prior to rural relocation where many small rural communities may not offer employment opportunities. $[14,30,48,53]$ This may lead to unemployment or long commutes to larger centres for employment. As such, it has been shown that younger female doctors are more likely to move out of rural settings due to their spouses' work commitments.[48]

\section{Limited childcare and schooling options}

Another identified barrier is limited childcare and schooling options, which impacts IMG integration and desire to stay in a rural area.[14,24,29-31,33,53] Female IMGs may rely on childcare to fulfill their work commitments; however childcare facilities are often limited in rural communities. If employment or educational opportunities are obtained for a spouse or partner then childcare support becomes increasingly necessary. Likewise, those IMGs with school age families may desire to move from rural employment or be deterred from accepting rural employment. This may be due to a lack of acceptable educational opportunities, particularly for those entering secondary schooling $[14,24,39,53]$.

\section{Physical and social isolation}

A lack of infrastructure is another significant barrier in recruitment and retention. For example, shopping centres, public transport; social organisations to develop interests and basic amenities are needed to retain IMGs and their families.[42] A lack of infrastructure can impede the 
transition and retention of IMGs and families in rural communities. Stanley and Bennett [42], who discussed this issue, in their policy position statement on supporting doctors' families in rural and remote communities, state "In many situations, this represents a significant contributor to social and physical isolation". As such, isolation can be heightened if public transport is deficient and a spouse does not drive.

It has been reported that many IMGs and their families living and working in rural and particularly remote communities do encounter physical and social isolation. [30,33]Nevertheless, an IMG by the nature of their employment has interaction with both the community members within the work environment.[30,54] However, culturally and linguistically diverse (CALD) individuals may also find language barriers in the workplace and community, which can further increase social isolation. In some cases, it was demonstrated in the Western Australian study conducted by Durey [34] that several IMGs and their families would travel hundreds of kilometres to participate in and visit with people from their same CALD community.[30,54]

Similarly, those IMGs from an Asian or Hispanic background in the US, were shown to have a greater propensity to seek employment where a greater proportion of similar CALD communities occur.[22] Thus, the availability of cultural activities and entertainment is a vital factor in IMG integration and retention.[14,27,48] Increased isolation can occur if contact with cultural, social or religious background is difficult or non-existent.[14,27] This hampers integration of the IMG, but more so their family.[42]

\section{Cultural and Religious Connectivity}

When isolation from cultural or religious background occurs, the maintenance of cultural and religious values, as well as connectivity with the respective CALD community was viewed as highly important within US, Australia and New Zealand studies.[14,22,25,26,30] Carlier, et al. [26] stated providing information about how to obtain cultural foods; linking IMGs with the same cultural or religious background; and providing a local mentor for family support assisted integration. However, elsewhere IMGs and their families found the move to rural life a little more challenging due to cultural, language and religious differences and a lack of extended family or friends.[25,30]

\section{Social Support}

To aid social isolation, social support for IMGs, their family and engaging in community participation are also essential elements to ensure integration occurs in rural communities.[27,51] The probability of and capability to integrate within rural communities has been shown to be an element of professional satisfaction irrespective of an IMGs cultural and religious background.[28] In addition, as social support facilitates the integration of IMGs and their families into the community, while discrimination and other obstacles can accelerate a family leaving.[27,28]

It was demonstrated by Atri et al. [16], in their US study, social support aids mental health and wellbeing, which in turn assists "in successful cultural adaptation and, eventually, in the optimal integration of IMG residents into the U.S. physician workforce". Thus, by addressing prevailing barriers through community orientation and knowledge of IMGs cultures and difference can assist a community's empathy and consideration for IMGs their spouse and children.[27]

Community orientation can assist integration within the community, whereas, indifference, cultural stigma and judgmental discourse can create lasting and profound apprehension and anxiety.[27] For example, it was shown in an Australian study, which looked at community integration in rural communities, that "some patients are reluctant to consult [IMGs], both in the hospital emergency room and the practice setting... [however] rural people are more cautious than their urban counterparts in what they say to doctors in general conversation.[27]

In addition, social and community events also meaningfully aided the acceptance, wellbeing and a sense of belonging for IMGs and their families.[26,28] When first migrating, IMG experience a loss of personal identity, social status and a sense of belonging. [16,17,23] Thus, these types of community connections assist IMGs and their families to feel a sense of belonging and support, which reduces the cultural dislocation they experience from family and friends.[30,48] In addition, those IMGs and families who actively engaged and participated in the community, through school activities, voluntary work, community events and social outings, had a greater acceptance by the community and community integration.[26,28]

\subsubsection{Overview of literature}

A review of the literature has indicated previous IMG studies have focused primarily on acculturation and retention issues within the workplace, such as IMG satisfaction and practise support.[7,24,26-30,45]Only a small number of these studies recognised quality of life and psychosocial needs of IMGs and their families as a crucial. factors impacting rural acculturation and retention $[42,53,55]$ As such, Alexander and Fraser [24] stated IMGs non-professional needs in rural contexts required further research. While Klein, et al [15] have stated there is a need to undertake further qualitative research to understand what motivates IMGs to stay in a certain place.

\subsection{Tasmania}

Tasmania is a small island state off the south east coast of mainland Australia with population over $\mathrm{n}=500,000$ [56-58], which has three area health services which each contain a major public hospital. This includes the Royal Hobart Hospital (RHH) in the South, Launceston General Hospital (LGH) in the North and the North West Regional Hospital (NWRH) in Burnie which incorporates the Mersey Hospital 
campus in Latrobe in the North-West of Tasmania. [59] As such, IMGs constitute approximately up to $30 \%(n=350)$ of registered medical practitioners in Tasmania which represents $2.57 \%$ of IMGs working across Australia, however the exact number of IMGs in Tasmania is unknown. [4,60,61] Nevertheless, General Practice Tasmania conducts an annual census which showed in 2011 there were a known $n=176$ General Practitioners (GP) practicing in Tasmania who were IMGs. [61] In addition to working in General practice, IMGs work in various other capacities such as hospital interns, registered medical officers, registrars and both public and private consultants.

In addition to the limited demographic data of IMGs in Tasmania, there is limited knowledge and research concerning the acculturation process of these IMGs as they reside in Tasmania, a less culturally diverse region of Australia. [62,63] As outlined, previous research on IMGs in other regions of rural Australia has focused primarily on employment integration, satisfaction and practice support as a measure of acculturation and retention. [29,30,62,64-67] Only a small number of these studies recognised quality of life and social needs of IMGs and their families as crucial factors impacting acculturation. [42,55,68]

It is vital for IMGs to be part of and become integrated within all communities and to feel engaged with and valued by communities. IMGs and their families can undergo a substantial displacement in moving to rural and remote Australia including social isolation and financial adversities. The success or failure of IMGs in rural communities is linked with the quality of planning IMGs receive in addition to support throughout the whole process. $[7,42,48,69]$ It has been duly noted "rural doctors are also human beings, requiring appropriate facilities to meet their quality of life needs". [68] Lastly, having sufficient support for the family of an IMG is essential to ensure they are strengthened by and integrated into the community. [7,42,48,69]

\subsection{The Context of the Study: Lost in the Labyrinth}

Within ten months of commencing the current study, 'Lost in the labyrinth', an extensively detailed Parliamentary inquiry on IMGs was published in March 2012. [4] The inquiry was conducted by the Standing Committee on Health and Ageing between December 2010 and March 2012, where 184 submissions were received from organisations, government bodies and individuals. [4] In addition, twenty two public hearings were held between February 2011 and January 2012 to gather further verbal evidence. The objective of the inquiry was to explore the registration process and support available for those [IMGs]... and to explore ways to remove impediments and promote pathways for [these] doctors to achieve their full Australian qualification, particularly in regional areas, without lowering the necessary standards required by colleges and regulatory bodies. [70]

The information of the inquiry was provided by many key informants, peak bodies, organisations and individuals across Australia. [4]From the Parliamentary inquiry, it was noted in there was support and provision made for the social needs of IMGs and their families in Tasmania. [70] Despite this, very little research is given to the integration and acculturation of IMGs as they reside in the rural Tasmania context. Thus, it is within the context and in light if the Parliamentary inquiry that the study was conducted.

\section{Objective}

This paper reports on the initial stage of a larger Doctor of Philosophy $(\mathrm{PhD})$ study which had no external source of funding or data sharing. As such, the objective of this initial stage was to investigate from the perspective of IMG informants what challenges and barriers are faced by IMGs as they work and live in Tasmania. IMG informants, such as medical educators, directors of clinical training and recruitment staff, were selected to participate in the study due to their expertise and knowledge working with Tasmanian IMGs. It was anticipated they may have rich contextual information which will add to the researcher's depth of study. They understand and are aware of the key issues and challenges faced by IMGs who enter, work and live in the Tasmanian rural context. This includes the determining factors which cause IMGs to stay or leave Tasmania.

The opportunity to have contact with IMG informants also allowed the researcher to gauge the contextual and political frameworks which operate in Tasmania and to explore the key issues that affect how informants see and perceive IMGs including the key factors which impact on their roles and responsibilities. These participants also provided information regarding the policy challenges which IMGs and the informants face. Their input was aimed to provide direction for the study and also to improve future IMG policy both locally and nationally.

\section{Methods}

This initial stage of the study used qualitative research to investigate and explore the lived experience of IMG informants working with IMGs and their perception of the main issues faced by IMGs. [71-74]Gathering data from IMG informants was important due to their wealth of knowledge regarding IMGs, their specific challenges and needs within the Tasmanian context. [75]Many other comparable studies of IMGs have been framed by a number of theories without great consensus over one specific approach. Therefore, phenomenology was employed as the framework to understand the data and guide the study. Phenomenology was selected as it is the study of everyday subjective experiences of the lived world from the perspective of the person who is having the experience.[76-78] Put simply, "phenomenology [is] the individual's construction of a life-world".[77]Thus, phenomenology is the means to provide "rich, in-depth 
understandings about social phenomena in their natural settings, which cannot be captured by quantitative methodologies".[17] Its purpose is to allowa researcher to borrow "people's lived experiences so that the researcher can better understand the meaning or the significance of the event".[79]Thus, phenomenology is used to generate methods of understanding, which views world of lived experience as a fundamental source of research. [80,81]

Data were collected through semi-structured interviews with a purposive snowball sample of twenty-three $(n=23)$ IMG informants in Tasmania. Informants were initially recruited from third party organisations that assist IMGs in either the acute care sector or general practice sectors of Tasmania. These organisations include the University of Tasmania, Department of Health and Human Services (DHHS), General Practice Tasmania Limited (now Tasmanian Medicare Local), General Practice Training Tasmania (GPTT), Health recruit plus (HR+) and Post Graduate medical council of Tasmania (PMCT) with further snowball sampling occurring once initial contact was made.

Informants were identified as key individuals who recruit, support and act as educators and advisors to IMGs in the Tasmanian public and private health workforce.[75] They include those who were in clinical and non-clinical backgrounds who worked full or part time in various capacities such as medical educators, directors of clinical training, program officers, organisational heads and recruitment management and staff. Due the nature of the snowball sampling, new informants were identified as original informants were being interviewed. Many newly identified informants were contacted and subsequently interviewed.

Each participant was given a copy of the questions and an information sheet before taking part in the study and prior to giving informed consent. Participants were interviewed once in English by one researcher. The Interviews ran between 20 and 75 minutes and were conducted between September and December 2011.The interview questions used in the this study were based a Victorian study, "The Retention of Overseas Trained Doctors in General Practice in Regional Victoria", conducted by Hawthorne, et al. [29] The Victorian study interviewed key informants to understand the perceived factors likely to influence IMG retention in general practice in rural Victoria and were focused on rural retention issues, including professional and social support issues.

The interview questions for this study consisted of questions relating to demographic background, the participant's role and experiences working in relation to IMGs in their location. This includes questions relating to the informant's own observations regarding the professional and social transition challenges typically faced by IMGs and what services are available to aid IMGs. For example, 'What type, level and adequacy of professional or social support services are provided to IMGs in this location?' and 'What are the professional transition challenges typically faced by
IMGs in this location?' The final questions related to what services are available to IMGs and what could be done to assist IMGs settle into rural communities and improve IMGs health and wellbeing in Tasmania. For example, 'Is there anything that you would like to see in the future to assist IMGs settle into rural communities in Tasmania?' and 'In your view, what should be done to improve IMGs health and wellbeing in Tasmania?'

More in-depth questions were asked throughout the interview to gain greater insight into each informants lived experience of working with IMGs and how IMGs from, their perspective, were adapting to rural Tasmania. This includes issues or concerns which may have been raised, for example, 'If you are asked to give advice to IMGs or colleagues on how deal with social or professional problems, what would you tell them?' Throughout each of the interviews clarification and probing questions were asked, which elucidated further information about particular IMG topics, which the participant had raised and was independent from the interview schedule.[76]In addition to the interview questions and the flexibility of discussion within the interviews, reflexivity and refinement of the interview schedule also occurred as interviews were conducted.[77] At the conclusion of each interview a reflexive journal entry was made. This allowed the researcher, who is not an IMG, medical practitioner, informant or sociologist, to write the views, feelings and impression gained from the participants. This reflexive process also allowed the researcher to highlight and review his own role and any biases which may have arisen throughout the research.

Ethical approval for the research was obtained from the Social Sciences Human Research Ethics (Tasmania) Network. The interviews were voice recorded, transcribed, coded and analysed by one researcher using QRS-NVivo v9.0 software. [82] For the purpose of the initial study, thematic analysis was used to identify recurring themes, patterns of living, behaviour and experience which then become a description of phenomenon. This also includes newly identified or emerging themes. [11,83,84]It was anticipated the qualitative data would illuminate the issues and problems faced by IMGs and their immediate families in rural Tasmania who are experiencing employment and social challenges.

\section{Results}

The twenty-three informants include those with clinical (45\%) and non-clinical (55\%) backgrounds who worked full or part time in various capacities as outlined in Table 1. It must be noted; two of the twenty-three informant's interviewed were also IMGs, yet were working as an organisational leader and a director of clinical training. Thus, these two informants were included in the sample, as they both had lived in Tasmania for a significant amount of time $(<5$ years) and had substantial contact with a large proportion of IMGs across the state. 
Informant discussion was framed by their experience with and narratives of IMGs who they worked closely with. The lived experience of informants was encompassed by and portrayed through the experiences they had with IMGs. This became more evident when points of view expressed by informants, particularly those with very close contact with IMGs, were echoing the points of view and the experiences expressed to them by IMGs. In this sense, the informants in the study became a conduit to the lived the experience of IMGs themselves. These informants, in many cases were an IMGs principal contact if there was a concern, issues or excitement occurring in their lives, such as passing an examination or the birth of a child.

Table 1. Characteristics of informant participants

\begin{tabular}{ccc}
\hline Characteristics & $\begin{array}{c}\text { Number of } \\
\text { participants }(\mathrm{n})\end{array}$ & $\begin{array}{c}\text { Percentage } \\
(\%)\end{array}$ \\
\hline $\begin{array}{c}\text { Clinicians } \\
\text { Staff working in acute } \\
\text { sector } \\
\text { Years of experience }\end{array}$ & 11 & $45.0 \%$ \\
Less than 2 years & 13 & $54.0 \%$ \\
2 to 5 years & 2 & $8.7 \%$ \\
More than 5 years & 10 & $43.5 \%$ \\
\hline Total & 11 & $47.8 \%$ \\
\hline
\end{tabular}

The study identified a number of key themes, which were framed around IMGs length of stay within Tasmania. As such, the data reflects much of the known literature, where length of stay is dependent upon a myriad of complex conditions related to the professional and social needs of each individual IMG and their family. [29] In addition there are a number of newer themes which have also arisen through the data and are under two broad themes, professional and social challenges, which are discussed in detail.

\subsection{Professional Challenges}

The professional challenges discussed by IMG informants were defined under four main themes. This includes differing medical systems, current workplace challenges, meeting future professional challenges and meeting cultural needs within the workplace. Also highlighted was the current professional support available for IMGs. Workplace challenges and professional support featured predominantly within each of the interviews. However, this was anticipated, as the main focus and obligation of many of IMG informants is being aware of, addressing workplace challenges and providing professional support.

\subsubsection{Differing Medical Systems}

Alternate and unfamiliar medical systems, models of care and health conditions are a major challenge to many IMGs, particularly those from less developed or western influenced countries. Concern was raised regarding IMGs who have come from medical systems where there may have inadequate or poor training or experience due to a lack of resources and equipment. In addition, IMGs who come from diverse cultural and religious mores were reported to have difficulties adjusting to a new discourse of care such as, patient-centre care and multidisciplinary work. Four (17.5\%) informants also raised the issues which some IMGs had with a number of common western health conditions which they were unfamiliar with. For example, sexually transmissible infections (STI), chronic disease and even diagnosing chicken pox due its presentation on a less familiar skin colour. However, it was reported the skills which a number of IMGs bring as they migrate were seen as a noteworthy addition to local skills. This positive was observed to be advantageous to accommodate today's migrating population, where one-quarter of Australia's population born overseas. [52]

\subsubsection{Workplace Challenges}

In addition to becoming familiar with a new medical system, models of care and health conditions, there were other workplace challenges reported by informants, to affect IMGs. For example, one of issues raised was the stress which IMGs encounter within the workplace. It was felt the major contributor of stress was caused by the unfamiliar, stress to perform, stress regarding job security, and stress caused by feeling professionally isolated. These stressors were felt to emanate from significant workplace causes, such as vulnerability to exploitation, racial intolerance and discrimination. It was also reported, hierarchy, power and communication within acute and primary care also being cited as key challenges.

\section{Vulnerability}

A large proportion of informants felt IMGs, were being used unfairly in the workplace. For example, it was reported by informant 4 , IMG interns and residents were being asked to work extra shifts, do extra hours and thus were reported as being overloaded. Intrinsically, IMG vulnerability was observed to be caused by fear of job security and at the heart of the stress they were experiencing. This was leading to tiredness, 'burn-out' and fatigue. While, the loss of status was also observed to be a cause of IMG vulnerability from less confidence within themselves, but it was also felt to be fuelled by other medical professionals. These other health professionals were observed to exhibit less respect for IMGs and viewed them as a "hostage workforce" (Informant 13).

\section{Discrimination and racial intolerance}

In addition to being viewed as a hostage workforce, over half $(n=12)$ of the informants stated there were elements of discrimination and racial intolerance, which often occurred in small pockets and made IMGs uncomfortable and stressed. This occurred when IMGs spoke with strong accents or spoke quickly and impact on being understood and communicating effectively with other health professionals. 
In comparison to how IMGs were reported to be treated a decade ago, five (21.7\%) of informants stated tolerance and understanding had vastly improved amongst those working with IMGs in Tasmania. However, informants did report there was still a perpetuating view among peers and supervisors of IMGs that these IMGs should better "assimilate" (Informant 1). Conversely, one informant argued "there was not a culture of racism or discrimination within the health system, but rather a culture of tolerating it" (Informant 13).

\section{Communication challenges}

Communication according to all informants was the most vital and greatest challenge faced by IMGs in the workplace. This extended from communication between colleagues as well as to patient in terms of comprehension. It was reported by informants; patients and colleagues often did not understand IMGs when communicating with them. In addition, a number of IMGs were having difficulty understanding their peers, and subsequently being perceived as rude or uncooperative. Communication skills workshops to assist with colloquialisms, vernacular and cultural nuances were a high priority throughout the various IMG informant organisations, with significant resources available to combat this issue. However, due to competing workplace demands and a lack of self-awareness of IMGs poor language skills, improving this challenge was reported to be problematic.

\subsubsection{Future Professional Challenges}

The current financial climate was also observed to be having a professional impact on IMGs. With the recent increase in domestic commonwealth-supported medical students, the future for IMGs in Tasmania was felt to be unclear. Many informants were speculating that the political context of IMGs practicing in rural Australia was about to dramatically change for many doctors who were in Australia already, and also for those contemplating migration to and within Australia. One informant stated IMGs "are academically brilliant" (Informant 8), but due to increased competition and the disadvantage of communication skills, they would find obtaining specific training and employment challenging to near impossible due to a potential poorer interview performance. In addition, another informant reported preferential selection process for non-IMGs was occurring already. With these changes and the potential for employing less IMGs, there was no real incentive for local hospitals to improve or provide greater assistance to meet a number of the professional challenges faced by current IMGs.

\subsubsection{Meeting Cultural Needs within the Workplace}

Four (17.5\%) informants made reference to the need to meet the cultural needs of IMGs within the workplace. They argued that rather than making IMGs out rightly 'assimilate', a greater cultural awareness must be proactively used within the healthcare profession. They felt it was about being more inclusive by recognising differences and some cultural needs are required to be met for a greater harmonious medical community. The views shared by a majority of informants, particularly those of whom had very close contact with IMGs understood acculturation was a 'give and take' process and not one sided. For example, two informants discussed the provision of acceptable and non-offensive prayer space being provided with practicable access for IMGs. Also another discussed how she was integral in ensuring religious or culturally acceptable food was being made available in the hospital cafeteria after several IMGs had approached this informant about their concerns. This change in the availability of food was noted to have occurred with the instigation of a number of informants. Nevertheless, within the various hospital settings, there was a large variation in what was occurring in terms of being aware of and meeting the cultural needs of IMGs within the workplace.

\subsection{Social Challenges}

In addition to the professional challenges discussed by IMG informants, a number of predominant social challenges were highlighted. This included the availability of initial and ongoing social supports were available for IMGs as they migrate to Tasmania. Many of the informants stated it was the social challenges which had the greatest impact upon IMGs, particularly their families, which determined the longevity of staying in one place. Nevertheless, those individuals who wish to contribute to or be part of the community do so by participating in social activities within the community. This includes such things as sports, school activities or actively participating in volunteering. [85] This community participation was seen to provide increased opportunities for connection the wider community.

Nevertheless, when IMG informants discussed the social challenges and support, a greater delineation between IMGs in the acute care and general practice settings became evident. As such, it was apparent that IMGs within the acute care setting had a greater number social challenges and less official social support. However, these same IMGs had greater collegial support and networks to aid many of the social challenges they faced, but this was also dependent upon the acute care setting and the IMGs position or stage of training. For example, interns and residents received a greater amount of official pastoral support, whereas registrars and specialists were provided with greater collegial support. This structure and delineation within the acute care was viewed by informants to be reminiscent of the training structures in place for interns, residents and registrars.

In contrast, IMGs within the general practice setting were perceived, by informants, to have similar, yet a lesser number of social challenges, greater official support and a lower amount of collegial support in comparison to those IMGs in the acute care setting. However, it was revealed that informants believed that IMGs within the general practice setting had a greater connectivity to the community in which they lived and served, than what was observed by informants regarding IMGs within the acute care setting. Still the social 
challenges each IMG face are similar with a number of these challenges outlined below.

\subsubsection{Spouse Employment}

According to eight (35\%) informants, employment for the spouse is a concern for many IMGs and they found it was an essential factor for settlement success. A smaller number of informants' stated spousal employment was often dependent upon where in the state the IMG and their family were residing, the skills which the spouse possessed and the current economic climate. For example, it was more challenging for a spouse to find appropriate employment in many towns on the west coast of Tasmania. One particular spouse, who was a nurse, had to travel over 200 kilometers from the West Coast to a larger regional centre of Tasmania as it was the only suitable work she could find. In addition, many spouses were stated to be highly skilled, such as engineers and accountants and it was reported that while some had found work, many had not, and those who did not find work, took a long time to settle into the community.

\subsubsection{Education for Children}

The provision of good education for children was also highlighted along with appropriate employment for a spouse as a key social challenge within the interviews. It was noted that informants thought that children of an IMG parent were known to be reasonably resilient when attending school and making friends. An informant stated "I haven't heard many people say oh my kids are so depressed or aren't coping...[however] high school is a big challenge and it is more about getting their kids the best academic access" (Informant 20).

However, at least one family was reported as having moved from Tasmania to another state due to the children having issues with school. High school had been voiced by informants as a major concern for a number of IMGs. Primary school attendance was, on the most part, suitable for children in rural areas of Tasmania. When high school was required this became a major motivator for being in closer proximity to larger centres such as Launceston and Hobart. Many rural areas of Tasmania do not have high schools or particularly pre-tertiary centres, thus making a geographical move or boarding school necessary. Comparatively, two informants argued Australian Medical Graduates (AMG), who are medical practitioners who are trained in Australia, also faced with similar concerns and issues. The informants felt obtaining the best educational access was a major concern for all doctors in the rural areas of Tasmania. This was reiterated by informant 20 who felt rurality did not discriminate against nationality when it came to accessing education for children. This informant succinctly stated "most Australian doctors don't last long in these communities either because of the education. Their kids need to get to high school and they want to bring them into the city" (Informant 20).

\subsubsection{Cultural and Religious Connectivity}

Cultural and religious connectivity was also highlighted as another major social challenge among a number of IMGs, yet it was also seen to some degree to be dependent upon nationality. Most informants stated there were a number of cultural and religious communities, which IMGs tapped into and who feature prominently within the various larger centres of Tasmania. These include Indian, Sri Lankan, Pakistan and Burmese groups, who have strong social or religious grouping outside of the workplace. These cultural groups get together with their families, participate in activities and even provide child care amongst each other. In addition to the general cultural communities, there are cultural communities within many workplaces which aide the transition of new IMGs in Tasmania.

Most IMGs of the same nationality were cited as being drawn to one another and look out for each other. These hospital 'communities' or connections were felt, by informants, to be good for the initial transition into the hospital and Tasmania, however long term participation was felt to be inhibitory. One experienced informant recognised these connections, although good, may also impede cross cultural connectivity with colleagues and the larger hospital community. However, another informant observed those IMG GPs who were staying longer and settling down in rural communities tended to have greater connectivity with others from their nationality. These observations by six (26\%) IMG informants reflects Berry's [86] acculturation theory, where the acquisition or rejection of the receiving culture combined with the retention or rejection of the heritage culture will determine the level of acculturation which occurs among migrants. For example, acquisition of the receiving culture and retention of the heritage culture leads to integration and rejection of the receiving culture and retention of the heritage culture leads to separation. As such, within the study, IMGs who retained connections with those of the same cultural and linguistically diverse communities, yet did not acquire connection with the wider hospital community found being in Tasmania challenging.

Nevertheless, even with these 'larger' cultural communities, in close proximity, there are a number of IMGs and their families who still continue to move to mainland Australia. Informants spoke of their experience in seeing these IMGs and families move because they wanted to be in the larger cities or closer to family and friends and would often gravitate towards much larger and more supportive cultural and religious communities. It was argued that AMGs also find cultural support and stimulation in some rural areas difficult also because the location "is fairly stressful [and] you are just lacking that peer or cultural interest. You do have sports, but not so much of the arts and movies, so Australian doctors go out there for five years... and then come back" (Informant 20).

\subsubsection{Isolation}

Isolation was also observed by informants to be a social challenge, as many IMGs require a number of essential services. Phone access and transport were the only two 
barriers which were highlighted by informants as issues for IMGs within the Tasmanian context. As such, isolation occurs for many IMGs, particularly those in general practice when compared to the acute setting, as public transport is problematic or nonexistent in more rural areas. In addition to this, many IMGs were unable to drive or lacked the confidence to drive in Tasmania. This issue is of particular importance to IMGs given that they often lack means of transport which would enable them to participate more widely within the community. A small number of IMGs were reported by one informant, as being in serious accidents when returning home from training in one of the larger centres. Another informant, who was also an IMG, stated many female IMGs known personally to them were not able to drive as their culture is one where women do not drive in their home country.

In addition, recruitment issues were occurring as IMG GP registrars who cannot drive remain reluctant to take up rural or satellite postings. If they do, it was seen by informants that these IMGs become increasingly isolated and there is an anticipation they "are going to leave, or plan to leave" (Informant 21). In addition to these challenges, those GP registrars, who can drive, become frustrated due to the inequity in placements caused by those who are unable to drive.

\subsection{Factors Influencing Retention}

Informants were asked questions regarding possible things which could be done to improve retention in Tasmania. Responses to these questions were varied amongst informant participants. Some informants felt there was not a lot that could be done to retain IMGs as many IMGs saw Tasmania as a temporary place of residency. For example, it was believed that many IMGs were coming to Tasmania to pass the Australian Medical Council (AMC) examination, obtain registration with the objective of leaving Tasmania. For example, one informant stated that:

[IMGs are using] Tasmania as a stepping stone to somewhere else... We get people here who just getting a foot in the door and they basically arrive here looking for greener pastures. That is a really big problem for us because they are expensive to recruit, they don't really want to be here, they tend not to perform particularly well... and they tend to milk the system. (Informant 8)

In many cases, Tasmania was stated to be seen as less attractive than other states such as Victoria, if unsuccessful in gaining employment. This was seen to be due to the geographical proximity and larger population size of Victoria, which was believed to provide greater access to family and friends who are living in Victoria and other states. Regardless of their motivations for coming to Tasmania, there were three main perceived factors, identified by informants which determined the retention of IMGs outside the professional and social challenges highlighted.These include, job opportunities and security, vocational training and greater IMG support.

\subsubsection{Job Opportunities}

Most informants stated if job opportunities or training schemes were available post AMC examination, IMGs would take them. As one informant commented in regards to job opportunities, many junior appointments are only twelve months and at times two year contracts are offered "once we know someone is committed" (Informant 1). It was argued that many IMGs, after receiving registration, do wish to stay in Tasmania, and have purchased houses and are settled, however leave the state due to a lack of available employment or specialist training. Nevertheless, it was stated many IMGs as with AMGs saw greater movement within Australia as part of their experience and training. So too, registrars rotate across states on a three, six or twelve monthly basis depending upon the position and the training they require. Additionally, it had been reported specialists were less likely to move once in Tasmania. "We don't lose a lot [of specialist]; we only lose those who have to go for their training" (Informant 6).

\subsubsection{Vocational Training}

Often it is the training requirements and opportunities which dictate where registrars can go for work and the length of time they reside in a particular place. However, there have been reports of a few IMGs who have left Tasmania for further training and have returned "because this is their home and this is where they want to be" (Informant 6). However, this is a phenomenon which also occurs among Tasmanian AMGs.

Moreover, training positions are becoming increasing competitive due to increased domestic production of AMGs. The increased graduation of AMGs has occurred since the announcement, in 2010, to spend $\$ 632$ million over 10 years to train more than 5,000 new doctors in Australian medical schools. [87,88] With the increased number of Australian trained medical students it is anticipated many new interns, including IMGs may not be guaranteed a placement to complete supervised training, which has raised as a particular concern in Tasmania. $[89,90]$

In addition, elements of vocational training are less or not available in Tasmania or are being lost. This loss was expressed to be due to the inability to retain college accreditation as a result of the diminishing of the service delivery in Tasmanian hospitals. As one informant argued "specialists are leaving, wards are getting smaller, we don't have the patient load, and they are not seeing the cases that they are meant to see for their curriculum, so they are getting de-recognised" (Informant 21). Therefore, a number of significant positions are at risk of being lost, this in turn was seen to be perpetuating the movement of IMGs from Tasmania as they returned to their country of origin, moved interstate or changed career pathways.

Also many training programs, such a general practice, require IMGs to have permanent residency visas before they can be accepted on the program. This has been inhibitory as other training programs, such as emergency medicine, which 
does not require this. Therefore it was reported many IMGs with families who would like to stay in Tasmania and commence general practise training are moving. This is so they can commence training in other fields and complete much of their training while still waiting for permanent residency.

\subsubsection{Greater IMG Support}

In addition to the employment and training opportunities, many informants noted it was the professional and social challenges of IMGs and their families which played a large part in the longevity of staying in place. Eight $(35 \%)$ informants restated if greater supports were in place, this may also improve IMG retention. One informant commented IMG retention rates had increasing since improved support for IMGs was developed. Other informants stated when speaking about the acute setting, greater consistency was required.

However, when speaking of IMGs working in general practises, improving IMG retention rates required face-to-face contact to provide skills training with greater information technology (IT) support to build education or clinical support networks. In addition, greater locum support was identified as a need to allow more time away from work. It was argued this was only half the solution, as there needed to be support provided to the family through greater and improved community engagement. These two improvements would require a substantial amount of funding and appropriate support.

Though, a predominant informant argued both IMGs and non-Tasmanian trained AMGs face similar challenges, such as difficulty acclimatising to a new health system when moving to Tasmanian. As such the informant stated what is needed is:

The same nature of support in place for everybody, which is then scalable depending on their identified need. What that requires is the infrastructure to provide it, the capacity to adequately identify and quantify the need, the wherefore to provide the assistance and the capacity to assess how effectively it has been and continually re-imagine and revise and refine the process. (Informant 13)

\section{Discussion and Recommendations}

Much of the qualitative interview data presented has highlighted workplace challenged which IMGs face, including vulnerability due to fear of job security, loss of status, discrimination and communication challenges within the workplace being a big concern of informants. This reflects the literature where a number of these challenges have been highlighted by informants in previous studies. $[29,49]$ This is particularly evident where a loss of status and professional identity [16,17]; professional isolation in rural practise; the need to sit further medical examinations, the restrictions on medical practise; language and communication proficiency; and accessing provider numbers occurs. [25,42,49] Furthermore, from the informant data, it was shown that incongruous placement of IMGs may impact negatively on the doctor, their family, the medical practice and the community. As such, this was similar to the literature where many difficulties and challenges are experienced at the time of recruitment. $[27,33,39]$ This may lead to an insufficient understanding of rural communities, how medicine is practised in Australia and how the Australian culture is at times vastly different to an IMGs own culture and experience. [27,33,39,40]

In addition, a community's limited experience with migrants coupled with the media scrutiny of Dr. Jayant Patel, an IMG working in Queensland, Australia. Dr. Patel, was implicated with 87 deaths occurring at the Bundaberg Base Hospital in Queensland between 2003 and 2005. [40,91-93] These challenges have restricted migrant acceptance within communities, where antagonism, discrimination and rejection produce poor long term acculturation, quality of life and poorer health of migrants. [19,27,33,39,40,44,94,95] In particularly, this intolerance has also been observed toward IMGs from both the public and within the medical profession. [39,44] For example, a lack of assistance has been observed from the medical profession, who at times has been divided by the employment of large numbers of IMGs to abate the rural doctor shortage in Australia. [27,31,51] However, a number of contrasting and very positive experiences have been voiced by IMGs in Australia. $[30,50]$

In contrast to the professional challenges, the social challenges of IMGs in Tasmania were also discussed in detail and reflect much of the literature regarding the similar social challenges faced by IMGs throughout Australia and internationally. $[29,30,48,62,68,96]$ These studies looked at the motivations for an IMG staying or leaving rural practise and showed non-professional, social barrier had the greatest bearing on the decision of where to practise. [25-27,30,31,51] The most prominent non-professional social barriers include employment for spouse, limited schooling and housing options, physical and social isolation and limited cultural and religious access. [13]As such, many of the social challenges highlighted within this study, have been shown to be universal challenges which face a diverse number of mobile skilled workers and their families which impacts integration and retention. [48]The underlying foundation of these social challenges was identified to be isolation.

The insights gained from the current study have shown the fundamental anxieties expressed by IMGs within the workplace and observed by IMG informants. These insights may provide further understanding for a number of challenges which IMG informants observed and experience when working with and assisting IMGs elsewhere in Australia and internationally. It draws attention to the fact that underlying the immigration, accreditation, registration and financial challenges IMG experience, there are other significant challenges, such as stress, vulnerability and isolation of both an IMG and their family, which may 
influence retention, acculturation and the ability to function effectively within the workplace and the community.

Nevertheless, amongst these challenges and concerns which were being shared by informants, many positives were also highlighted. This includes the professional and social support systems, structures and mechanisms which were in place to aide IMGs. It was noted, however, there were areas of improvement, such as increased funding and consistency between acute settings. It was observed by the acute sector that the general practice recruitment and support sectors were setting much of the standards with aiding IMGs both professionally and socially. An acute care informant stated it was this example, the general practice sector was setting, which the acute care sector could learn and emulate to provide improved services. Improvements had been observed over the past decade; however it was felt there was more that could be done.

In addition, a myriad of complex conditions were shown to occur which influenced length of stay and retention of IMGs within Tasmania. This is also impacted by a contracting of the health services within Tasmania, due to the current financial climate [97], and the limited training opportunities for certain career pathways. Therefore the emphasis on IMG retention needs to be developed and reworked. It is recommended that retaining IMGs within Tasmania requires a greater focus on promoting and improving the attractiveness and accessibility of those career pathways which are available and needed in Tasmania. As well as greater support to meet the identified professional and social needs of IMGs living throughout Tasmania, which could extend to AMGs in more rural and remote regions. It is however important to recognise the informant data presented has allowed the opportunity to gauge the contextual and political frameworks which operate in Tasmania and to explore the key issues that affect IMGs. Nevertheless, this data requires further research to be conducted to gather data from IMGs and their own experiences in Tasmania. This is the next planned stage of the larger doctoral study being conducted.

\section{Limitations}

There are a number of limitations to the study. It was recognised those informants who agreed to participate may be dissimilar to those informants who elected not participate in the study. Moreover, there may be issues of recall bias of those informants interviewed, as eleven had been informants for more than five years. In addition, two informants were IMGs, which may have introduced an element of bias within their views of the issues discussed in the interview. They had the potential to express their own experiences rather than the experiences of others. In addition, the views, experiences and data of the informants interviewed may not be transferrable or generalisable to all IMG populations. However, the sample methods used in the study were convenience, purposive snowball of a heterogeneous population. As such, these sampling methods were used to guarantee a representative sample from the finite population was collected. This ensured the results of the study sample could be more generalisable. [98] Nevertheless the study was intended to investigate what is occurring in less culturally dense communities such as Tasmania where paucity of research and data regarding IMG acculturation, health and wellbeing exists. The data provides a platform and highlights a number of key issues which will require more in-depth investigation in the future and in other rural contexts of Australia.

\section{Conclusion}

This study has examined the views, knowledge and understandings of twenty-three IMG informants in Tasmania who assist and support IMGs, a heterogeneous group of doctors, which encompasses a large number of nationalities, cultures and religious backgrounds. These key informant interviews have demonstrated a number of the existing professional and social challenges faced by IMGs. Much of which reflects the current data regarding IMGs across Australia and internationally. In addition, the informants have highlighted a number of identified needs to improve retention of IMGs within Tasmania. The study adds to existing knowledge by highlighting three identified factors which informants believed would further determine the retention of IMGs. These factors include, job opportunities, which is reliant upon current need, availability and economic factors; vocational training, which depends upon career pathway choice, availability and strength of competition; and finally it also depends upon the availability of commensurate support provided to IMGs and families. As such it requires a clearly identified and delineated need, the economic support and greater uniformity and co-ordination to ensure not only the professional but also the social needs of IMGs and their families are met.

\section{Acknowledgements}

The research team is most grateful to Dr Jess Woodroffe, Dr Kath Ogden and the participants of the study for their invaluable contribution. We would like to express our deep appreciation to University of Tasmania; Department of Health and Human Services; Tasmanian Medical Local; General Practice Training Tasmania; Health Recruit Plus; and Post Graduate medical council of Tasmania for their support in conducting the research.

\section{REFERENCES}

[1] Dauphinee WD. The Circle Game: Understanding Physician Migration Patterns Within Canada. Academic Medicine. 


\section{6;81:S49-S54.}

[2] Organisation for Economic Co-operation and Development. International Migration Outlook 2007. Danvers, MA: Organisation for Economic Co-operation and Development, 2007.

[3] Australian Institute of Health and Welfare. Medical Labour Force 2009. Canberra: Australian Institute of Health and Welfare, 2011.

[4] House of Representatives Standing Committee on Health and Ageing. Lost in the Labyrinth: Report on the inquiry into registration processes and support for overseas trained doctors. In: House of Representatives SCoHaA, editor. Canberra: Department of House of Representatives Printing and Publishing Office; 2012.

[5] Department of Health and Ageing. Work as a Doctor in Australia. 2011 [updated 2/02/2009; cited 201129 June ]; Available

from: http://www.doctorconnect.gov.au/internet/otd/Publishing.nsf /Content/work-RWAs-2.

[6] Laurence C. Overseas trained doctors in rural and remote Australia: Do they practise differently from Australian trained doctors? [PhD]. Adelaide, South Australia, Australia: University of Adelaide 2008.

[7] Rural Health Workforce Australia. Parliamentary Inquiry into Overseas Trained Doctors. Melbourne: Rural Health Workforce Australia, 201111 February. Report No.: 1.

[8] Australian Medical Council. International Medical Graduates Home Canberra: Australian Medical Council; 2012 [updated 29 April cited 20121 July]; Available from: http://www.amc.org.au.

[9] Australian Medical Council. AMC submission to House of Representatives Standing Committee Inquiry into Registration Processes and Support for Overseas Trained Doctors. Canberra: Australian Medical Council, 2011 Contract No.: Submission No 42.

[10] Australian Medical Council. Workplace Based Assessment resource guide. Kingston, ACT: Australian Medical Council; 2009.

[11] Fereday J, Muir-Cochrane E. Demonstrating rigor using thematic analysis: A hybrid approach of inductive and deductive coding and theme development. International Journal of Qualitative Methods. 2008;5:80-92.

[12] Department of Immigration and Citizenship. Visas, Immigration and Refugees. 2012 [cited 20121 July]; Available from: http://www.immi.gov.au/skilled/skilled-wor kers/sbs/.

[13] Terry D, Lê Q, Woodroffe J, Ogden K. The quality of life and social needs of International Medical Graduates: Emerging themes in research. International Journal of Annotated Interdisciplinary Research. 2011;1:59-67.

[14] Kearns R, Myers J, Adair V, Coster H, Coster G. What makes 'place' attractive to overseas-trained doctors in rural New Zealand? Health \& social care in the community. 2006;14:532-40.

[15] Klein D, Hofmeister M, Lockyer J, Crutcher R, Fidler H. Push, pull, and plant: The personal side of physician immigration to Alberta, Canada. Fam Med.

2009;41:197-201.
[16] Atri A, Matorin A, Ruiz P. Integration of International Medical Graduates in U.S. Psychiatry: The Role of Acculturation and Social Support. Academic Psychiatry. 2011;35:21-6.

[17] Wong A, Lohfeld L. Recertifying as a doctor in Canada: International Medical Graduates and the journey from entry to adaptation. Med Educ. 2008;42:53-60.

[18] Lillis S, St George I, Upsdell R. Perceptions of migrant doctors joining the New Zealand medical workforce. Journal of the New Zealand Medical Association. 2006;119:46-54.

[19] Mpofu C. Immigrant medical practitioners' experience of seeking New Zealand registration: A participatory study [Masters]. Auckland, New Zealand: Auckland University of Technology; 2008.

[20] Wawdhane S, Saraf V, Davidson S, Trewby P. Clinical attachments: Fond farewell or new beginning? A survey of the attitudes and practice of medical consultants and international medical graduates. Postgrad Med J. 2007;83:196

[21] Crouse BJ, Munson RL. The Effect of the Physician J-1 Visa Waiver on Rural Wisconsin. Wisconsin Medical Journal. 2006;105:16.

[22] Polsky D, Kletke PR, Wozniak GD, Escarce JJ. Initial practice locations of International Medical Graduates. Health Services Research. 2002;37:907-28.

[23] Shuval JT. The reconstruction of professional identity among immigrant physicians in three societies. Journal of Immigrant Health. 2000;2:191-202.

[24] Alexander C, Fraser J. Education, training and support needs of Australian trained doctors and international medical graduates in rural Australia: A case of special needs. Rural and Remote Health. 2007;7.

[25] McGrath P, Henderson D, Phillips E. Integration into the Australian health care system Insights from international medical graduates. Aust Fam Physician. 2009;38:844-8.

[26] Carlier N, Carlier M, Bisset G. Orientation of IMGs: A rural evaluation. Aust Fam Physician. 2005;34:485.

[27] Han GS, Humphreys JS. Overseas trained doctors in Australia: Community integration and their intention to stay in a rural community. Australian Journal of Rural Health. 2005;13:236-41.

[28] Han GS, Humphreys JS. Integration and retention of international medical graduates in rural communities. Journal of Sociology. 2006;42:189.

[29] Hawthorne L, Birrell B, Young D. The retention of overseas trained doctors in general practice in regional Victoria. Melbourne: University of Melbourne, 20030958183589.

[30] Durey A. Settling In: Overseas Trained GPs and Their Spouses in Rural Western Australia. Rural Society. 2005; 15:38-54.

[31] Gilles MT, Wakerman J, Durey A. If it wasn't for OTDs, there would be no AMS": Overseas-Trained Doctors working in rural and remote Aboriginal health settings. Australian Health Review : A publication of the Australian Hospital Association. 2008;32:655-63.

[32] Laven GA, Beilby JJ, Wilkinson D, Mc Elroy HJ. Factors associated with rural practice among Australian-trained 
general practitioners. Medical Journal of Australia. 2003;179:75-9.

[33] Durey A, Hill P, Arkles R, Gilles M, Peterson K, Wearne S, et al. Overseas trained doctors in Indigenous rural health services: Negotiating professional relationships across cultural domains. Australian and New Zealand Journal of Public Health. 2008;32:512-8.

[34] Durey A. The changing face of rural general practice: An ethnographic study of general practitioners and their spouses $[\mathrm{PhD}]$. Perth, Western Australia, Australia: Edith Cowan University; 2005.

[35] Lê Q, Kilpatrick S. Vietnamese-born health professionals: Negotiating work and life in rural Australia. Rural and Remote Health. 2008;8:1-15.

[36] Brown R, Connell J. The migration of doctors and nurses from South Pacific Island Nations. Social Science \& Medicine. 2004;58:2193-210.

[37] MacKenzie R, Forde C. The rhetoric of the good worker 'versus the realities of employers' use and the experiences of migrant workers. Work, Employment \& Society. 2009;23:14 2-59.

[38] Australian Rural and Remote Workforce Agencies Group. Policy Position Paper No. 2, "Overseas Trained Doctors within the Rural and Remote Workforce". Australian Rural and Remote Workforce Agencies Group, 2004 November. Report No.

[39] Han GS. International medical graduates in Australian news: A media narrative analysis. Journal of Health Organization and Management. 2010;24:237-57.

[40] Harvey K, Faunce T. A Critical Analysis of Overseas-trained Doctor (OTD) Factors in the Bundaberg Base Hospital Surgical Inquiry. Law in Context. 2005;23:73-90.

[41] Hagopian A, Thompson MJ, Kaltenbach E, Hart LG. Health departments' use of International Medical Graduates in physician shortage areas. Health Affairs. 2003;22:241-9.

[42] Stanley M, Bennett C. Supporting Doctors' Families in Rural and Remote Practice: Policy Position Statement. Carlton South, Victoria: Australian Rural and Remote Workforce Agencies Group 2005 October 2005. Report No.

[43] Maynard-Reid N. The relations among acculturation, acculturative stress, and social self-efficacy [PhD]. Arizona: Arizona State University; 2005.

[44] Louis WR, Lalonde RN, Esses VM. Bias against foreign-born or foreign-trained doctors: experimental evidence. Med Educ. 2010;44:1241-7.

[45] Heal C, Jacobs H. A peer support program for International Medical Graduates. Aust Fam Physician. 2005;34:277-8.

[46] Nair B, Parvathy M. Setting up International Medical Graduates to succeed. Medical Journal of Australia. 2012;197:428-9.

[47] Frehywot S, Mullan F, Payne PW, Ross H. Compulsory service programmes for recruiting health workers in remote and rural areas: Do they work? Bulletin of the World Health Organization. 2010;88:364-70.

[48] Kilpatrick S, Johns S, Vitartas P, Homisan M. Mobile skilled workers: Making the most of an untapped rural community resource. J Rural Stud. 2011;27:181-90.
[49] Wright A, Regan M, Haigh C, Sunderji I, Vijayakumar P, Smith C, et al. Supporting International Medical Graduates in rural Australia: A mixed methods evaluation. Rural and Remote Health. 2012;12.

[50] Lim CKD. Prescibing practices of Austrlian dispensing doctors $[\mathrm{PhD}]$. Perth, Western Australia, Australia: Curtin University; 2010.

[51] Hancock C, Steinbach A, Nesbitt TS, Adler SR, Auerswald CL. Why doctors choose small towns: A developmental model of rural physician recruitment and retention. Social Science \& Medicine. 2009;69:1368-76.

[52] Terry D, Woodroffe JJ, Lê Q, Ogden K. International Medical Graduates in Australia: A historical perspective (1930-1950s). Medical Journal of Australia. 2012;197:84-6.

[53] Alexander C. Why doctors would stay in rural practice in the New England health area of New South Wales. Australian Journal of Rural Health. 1998;6:136-9.

[54] Harding C, Parajuli N, Johnston L, Pilotto L. Comparing patients' perceptions of IMGs and local Australian graduates in rural general practice. Aust Fam Physician.2010;39:231-3.

[55] Colic-Peisker V. Visibility, settlement success and life satisfaction in three refugee communities in Australia. Ethnicities. 2009;9:175.

[56] Department of Health and Ageing. General Practice Workforce Statistics Canberra: Department of Health and Ageing; 2011 [cited 20117 June]; Available from: http://www.health.gov.au/internet/main/publishing.nsf/Conte $\mathrm{nt} /$ General+Practice+Statistics-1.

[57] Health Recruitment Plus Tasmania. GP Recruitment. 2011 [cited 2011 June 29th]; Available from: http://www.healthre cruitmentplus.com.au/gp-recruitment/choose-tasmania.htm.

[58] Australian Bureau of Statistics. National Regional Profile 2006-2010. Canberra: Australian Bureau of Statistics; 2012 [updated 2 November 2011]; Available from: http://www.ausstats.abs.gov.au/ausstats/nrpmaps.nsf/NEW+ GmapPages/national+regional+profile?opendocument.

[59] Department of Health and Human Services. Services by Area. Hobart: Department of Health and Human Services 2012 [cited 20128 July]; Available from: http://www.dhhs.tas.gov.au/.

[60] Department of Health and Human services. Personal communication. Hobart 2011.

[61] General Practice Tasmania Limited. Census of Tasmanian General Practitioners, October 2011. Hobart: 2011.

[62] Han GS, Humphreys JS. Overseas trained doctors in Australia: Community integration and their intention to stay in a rural community. Australian J Rural Health. 2005;13:236-41.

[63] Lê Q, Kilpatrick S. Vietnamese-born health professionals: negotiating work and life in rural Australia. Rural Remote Health. 2008;8:1-15.

[64] Alexander C, Fraser J. Education, training and support needs of Australian trained doctors and international medical graduates in rural Australia: a case of special needs. Rural Remote Health. 2007;7.

[65] Carlier N, Carlier M, Bisset G. Orientation of IMGs: a rural evaluation. Australian Fam Physician. 2005;34:485. 
[66] Han GS, Humphreys JS. Integration and retention of international medical graduates in rural communities. J Sociol (Melb). 2006;42:189.

[67] Heal C, Jacobs H. A peer support program for international medical graduates. Australian Fam Physician. 2005;34:277-8.

[68] Alexander C. Why doctors would stay in rural practice in the New England health area of New South Wales. Australian J Rural Health. 1998;6:136-9.

[69] Frehywot S, Mullan F, Payne PW, Ross H. Compulsory service programmes for recruiting health workers in remote and rural areas: do they work? Bull World Health Organ. 2010;88:364-70.

[70] House of Representatives Standing Committee on Health and Ageing. Registration processes and support for overseas trained doctors - Launceston hearing. In: House of Representatives SCoHaA, editor. Canberra: Department of House of Representatives Printing and Publishing Office; 2012.

[71] Bernard HR. Social research methods: Qualitative and quantitative approaches. 4th ed. Thousand Oaks: Sage Publications; 2000.

[72] Broom A, Willis E. Competing paradigms and health research. In: Saks M, Allsop J, editors. Researching health: qualitative, quantitative and mixed methods. London: SAGE Publications; 2007. p. 16-30.

[73] Calnan M. Quantitative Survey Methods in Health Research. In: Saks M, Allsop J, editors. Researching health: qualitative, quantitative and mixed methods. London: SAGE Publications; 2007. p. 174-97.

[74] Davis P, Scott A. Health Research Sampling Methods. In: Saks M, Allsop J, editors. Researching health: qualitative, quantitative and mixed methods. London: SAGE Publications; 2007. p. 155-73.

[75] Hawthorne L, Hawthorne G, Crotty B. The Registration and Training Status of Overseas Trained Doctors in Australia: Final Report. Melbourne: Faculty of Medicine, Dentistry and Health Sciences, The University of Melbourne, 2007 February 2007. Report No.

[76] Bowling A. Research Methods in Health: Investigating Health and Health Services. 2nd ed. Berkshire: Open University Press; 2005.

[77] Liamputtong P, Ezzy D. Qualitative Research Methods. 2nd ed. South Melbourne: Oxford University Press; 2005.

[78] Greenhalgh T. Primary health care: Theory and practice. Malden, Mass: Blackwell/BMJ Books; 2007.

[79] McConnell-Henry T, Chapman Y, Francis K. Unpacking Heideggerian phenomenology. Southern Online Journal of Nursing Research. 2009;9:6.

[80] Campbell B. Phenomenology as Research Method Victoria Univeristy of Technology; 2011 [cited 201118 May ]; Available from:http://www.staff.vu.edu.au/syed/alrnnv/pape rs/bev.html.

[81] Van Manen M. Researching lived experience: Human science for an action sensitive pedagogy. Albany, N.Y: State University of New York Press; 1990.

[82] QRS International. Nvivo 10.0. Melbourne: QRS Internation al Pty Ltd; 2012; Available from: http://www.qsrinternationa 1.com/\#tab you.

[83] Aronson J. A pragmatic view of thematic analysis. Qual Rep. $1994 ; 2: 1-3$

[84] Braun V, Clarke V. Using thematic analysis in psychology. Qual Res Psychol. 2006;3:77-101.

[85] Terry D, Terry M. Common interests, social ties and the development of social capital The Internet Journal of Language, Culture and Society. 2012:105-13.

[86] Berry JW. Immigration, Acculturation, and Adaptation. Appl Psychol. 1997;46:5-34.

[87] Elkin KJ, Studdert DM. Restricted career paths for overseas students graduating from Australian medical schools: Legal and policy considerations. Medical Journal of Australia. 2010;192:517-9.

[88] Rodgers E. PM pledges 5,000 new doctors. Canberra: ABC News; 2010 [updated 15 March 2010; cited 201130 September]; Available from: http://www.abc.net.au/news/20 10-03-15/pm-pledges-5000-new-doctors/364472.

[89] Caldwell A. Medical students still without internships. Canberra: ABC News; 2010 [updated March 17 2010; cited 201130 September]; Available from: http:/www.abc.net.au/ news/2010-03-17/medical-students-still-without-internships/ 368644 .

[90] Aizen M. Tasmanian turning away potential doctors. Media Release. Hobart: Australian Medical Association - Tasmania, 201022 July 2010. Report No.

[91] Moynihan R. Australia launches inquiry into treatment of Overseas Trained Doctors. BMJ. 2010;341:c6780.

[92] Birrell B, Schwartz A. Accreditation of Overseas Trained Doctors: The Continuing Crisis. People and Place. 2006; $14: 37-47$

[93] Birrell B, Schwartz A. Assessment of Overseas-trained Doctors: the Latest Chapter. People and Place. 2007;15:67-75.

[94] Crompvoets S. In search of an alternative discourse on International Medical Graduate issues. People and Place. 2010;18:27.

[95] Berry JW. Immigration, Acculturation, and Adaptation. Applied Psychology. 1997;46:5-34.

[96] Kearns R, Myers J, Adair V, Coster H, Coster G. What makes 'place' attractive to overseas-trained doctors in rural New Zealand? Health Soc Care Community. 2006;14:532-40.

[97] Giddings L. 2011-12 Budgest speech: 'Strong decisions. Better future.'. In: Treasury, editor. 16 June ed. Hobart: Tasmanian State Governement; 2011. p. 1-16.

[98] Marshall MN. Sampling for qualitative research. Family Practice. 1996;13:522. 